\title{
¿Es la evaluación ultrasonográfica del peso fetal influida por la fórmula seleccionada? (1)
}

\author{
Burd I, Srinivas S, Paré E, Dharan V, Wang E. J Ultrasound Med 2009;28(8):1019-24. \\ Análisis crítico: Victoria Pérez M. ${ }^{1}$, Jorge Carvajal C., $P h D^{2}$, Claudio Vera P-G., MSc ${ }^{2,3}$ \\ 1 Programa de Obstetricia y Ginecología, ${ }^{2}$ Unidad de Medicina Materno-Fetal, Departamento de Obstetricia y Ginecolo- \\ gía, ${ }^{3}$ Unidad de Medicina Basada en Evidencias, Facultad de Medicina, Pontificia Universidad Católica de Chile.
}

\section{RESUMEN (1)}

Objetivo: Existen varias fórmulas publicadas para la estimación de peso fetal (EPF), e información limitada de su rendimiento comparativo. El objetivo del estudio fue evaluar y comparar el rendimiento de las diferentes fórmulas de EPF en predecir el peso al nacer (PN) en una población urbana. Métodos: Pacientes con determinación de EPF dentro de los 7 días previos al parto fueron consideradas elegibles para este estudio. Catorce fórmulas publicadas, derivadas de población comparable a la nuestra, fueron usadas para recalcular la EPF usando las mismas medidas iniciales. El rendimiento de la EPF obtenidas desde las diferentes fórmulas fue comparado usando métodos de error porcentual de sesgo y precisión, y el método de límites de Bland-Altman para evaluar concordancia. Se calculó la sensibilidad y especificidad para predecir pequeños para la edad gestacional (PEG). Resultados: 81 fetos fueron incluidos en el estudio. La fórmula $\mathrm{C}$ de Hadlock et al $[\log 10 \mathrm{PN}=1,335$ - 0,0034(circunferencia abdominal)(longitud del fémur)0,0316 (diámetro biparietal) + 0,0457 (circunferencia abdominal) + 0,1623 (longitud del fémur); Am J Obstet Gynecol 1985,151:333-7] tuvo el mejor rendimiento de acuerdo al método de sesgo y precisión. El límite de concordancia de Bland-Altman confirmó estos resultados. Entre todas las fórmulas, la sensibilidad para detectar PEG varía entre $72 \%$ a $100 \%$, y la especificidad entre $41 \%$ a $88 \%$. Hadlock C tiene la compensación óptima para la detección de PEG. Conclusión: Las catorces fórmulas mues- tran una variación considerable en el sesgo y precisión en nuestra población, como también un amplio rango en la sensibilidad y especificidad para PEG. La elección de una fórmula apropiada para la EPF en una población determinada debe basarse en criterios explícitos y objetivos. Un examen de sesgo y precisión de la fórmula en la población que se está evaluando es crítica y puede afectar los cuidados clínicos.

\section{ANÁLISIS DE LA INVESTIGACIÓN}

\section{A. Relevancia clínica de la investigación}

Determinar cuál de las fórmulas para estimación de peso fetal ecográfico tiene mejor capacidad diagnóstica.

Escenario clínico: El peso al nacer es reconocido como una variable importante que afecta la mortalidad perinatal (2). Es por este motivo que la estimación de peso ecográfica tiene especial relevancia en la toma de decisiones clínicas tales como el momento de la interrupción y la vía de parto (3). Se conoce que las diferentes fórmulas para estimación del peso fetal tiene un margen de error cercano al $10 \%$, y que ninguna de las fórmulas utilizadas ha demostrado ser necesariamente mejor que las otras. Existe el concepto de que la mayoría de las fórmulas de EPF sobrestiman la presencia de bajo peso al nacer y al mismo tiempo subestiman el riesgo de macrosomia fetal (4). Existe limitada evidencia que compare el rendimiento entre diferentes fórmulas, lo que justifica la presente investigación. 


\section{B. El estudio (1)}

Objetivo: Comparar la capacidad de las diferentes fórmulas de EPF en predecir el PN en una población urbana. Pacientes: Datos de edad gestacional, peso al nacer y biometría (DBP, HC, AC, LF) de mujeres con EPF ecográfica dentro de 7 días previos al parto, obtenidos de pacientes de un estudio previo de caso control ("Preeclampsia: Mechanisms and Consequences"). Prueba estudiada: Catorce diferentes fórmulas ecográficas de EPF. Estándar de comparación: Registro del peso al nacer. Resultado primario medido: Evaluación de la fiabilidad a través de la comparación del error porcentual mediante el análisis de sesgo y precisión para cada fórmula y evaluación de la concordancia a través del método de Bland-Altman. Resultados: De las fórmulas evaluadas, Hadlock $\mathrm{C}$ tiene la mejor precisión y exactitud como también la mejor concordancia para predecir peso fetal en 81 fetos seleccionados. La formula de Hadlock C, que incluye circunferencia abdominal, longitud de femur y diámetro biparietal presentó un sesgo menor al $5 \%$ y precisión menor de $14 \%$ de la estimación de peso fetal. Con un rendimiento diagnóstico para pequeño para edad gestacional (PEG) expresado en sensibilidad de $76 \%$, especificidad de $88 \%$ y LR para PEG de 6.33, estimado con los datos publicados y calculado con CatMaker $\circledast$.

\section{Análisis crítico:}

Validez interna: Estudio que compara la concordancia y rendimiento diagnóstico de una serie de fórmulas para estimar el peso fetal. La población de origen incluye embarazos únicos de término (40 casos) y embarazos de mujeres portadoras de preeclampsia (41 casos) de un estudio caso control previo en un hospital universitario, que puede no ser necesariamente representativo de una población determinada. Se seleccionaron los casos que contaban con una ecografía antes de 7 días previos al parto. Todos los sujetos seleccionados contaban con evaluación biométrica ultrasonográfica que permitió el cálculo de EPF por distintas fórmulas y en todos se conoció el estándar de referencia (peso al nacer), la muestra contenía $28 \%$ de pequeños para la edad gestacional. La prueba de estudio y el estándar de referencia son independientes entre sí. Dado la naturaleza del estudio la estimación ultrasonográfica antecede al conocimiento del peso fetal, así los ultrasonografistas que realizaron las mediciones biométricas fueron ciegos al estándar de referencia. Se definió un criterio preestablecido para la comparación de precisión de las fórmulas (15\% del peso al nacer). Aplicabilidad: Los resultados de este estudio pudieran ser aplicables a embarazos únicos, de alto riesgo, que compartan características demográficas de la población en la que se realizó el estudio (Hospital Universiario de Pennsylvania). Es posible que variaciones en la proporción de pacientes de alto riesgo, proporción de fetos pequeños para la edad gestacional y configuraciones étnicas afecten el rendimiento diagnóstico de la fórmula. Comentario: Estudio con bajo riesgo de sesgo que evalúa la concordancia y rendimiento diagnóstico de diferentes fórmulas de estimación de peso fetal ultrasonográfica para predecir el peso al nacer. Su evaluación identifica una fórmula con mejor precisión y menor sesgo junto con mayor rendimiento para el diagnóstico de pequeño para la edad gestacional en una población seleccionada. Sin embargo, es importante hacer notar que variaciones en el espectro población estudiada pueden hacer variar el rendimiento diagnóstico de las fórmulas.

\section{BIBLIOGRAFÍA}

1. Burd I, Srinivas S, Paré E, Dharan V, Wang E. Is sonogaphic assessment of fetal weight influenced by formula selection? J Ultrasound Med 2009;28(8):101924.

2. Barker DJP. Long-term outcome of retarded fetal growth. In Clinical Obstetrics and Gynecology, Divon MY (ed.).Lippincott-Raven: Philadelphia, PA, 1997; 853-63.

3. Dudley NJ. A systematic review of the ultrasound estimation of fetal weight. Ultrasound Obstet Gynecol 2005;25:80-9.

4. Baker PN, Johnson IR, Gowland PA, et al. Fetal weight estimation by echo-planar magnetic resonance imaging. Lancet 1994;343:644-5.

5. Bland JM, Altman DG. Statistical methods for assessing agreement between two methods of clinical measurement. Lancet 1986;1(8476):307-10. 